\title{
Convergence of Laguerre Impulse Response Approximation for Noninteger Order Systems
}

\author{
Piotr Bania, Jerzy Baranowski, and Marta Zagórowska \\ AGH University of Science and Technology, Aleja Adama Mickiewicza 30, 30-059 Kraków, Poland \\ Correspondence should be addressed to Jerzy Baranowski; jb@agh.edu.pl
}

Received 29 April 2016; Accepted 3 July 2016

Academic Editor: Josè A. Tenereiro Machado

Copyright ( 2016 Piotr Bania et al. This is an open access article distributed under the Creative Commons Attribution License, which permits unrestricted use, distribution, and reproduction in any medium, provided the original work is properly cited.

\begin{abstract}
One of the most important issues in application of noninteger order systems concerns their implementation. One of the possible approaches is the approximation of convolution operation with the impulse response of noninteger system. In this paper, new results on the Laguerre Impulse Response Approximation method are presented. Among the others, a new proof of $\mathscr{L}_{1}$ convergence of approximation is given, allowing less strict assumptions. Additionally, more general results are given including one regarding functions that are in the joint part of $\mathscr{L}_{1}$ and $\mathscr{L}_{2}$ spaces. The method was also illustrated with examples of use: analysis of "fractional order lag" system, application to noninteger order filters design, and parametric optimization of fractional controllers.
\end{abstract}

\section{Introduction}

Noninteger order (fractional) systems are becoming more prevalent in control and signal processing applications. The fact that they cannot be directly implemented on digital or analog platforms is, however, a known issue. The issues come from difficulties with realization of noninteger derivatives and lack of semigroup property. The search for efficient approximation that will keep the beneficial properties of fractional system while allowing implementation is a topic of ongoing research.

In this paper, we discuss new developments in the Laguerre Impulse Response Approximation (LIRA) method. This method allows efficient approximation of wide class of noninteger order systems, in particular noninteger order filters. Those new developments include formal proofs of convergence in both general and particular cases and proofs of important properties needed for implementation.

Besides LIRA, there are also different methods of noninteger order system approximation. They can be divided into three groups. The first group is based on approximating $s^{\alpha}$ operator in the frequency domain. The most popular approaches are Oustaloup's method $[1,2]$ and CFE (Continuous Fraction Expansion) method [3-5]. These methods are based on different premises but both allow obtaining relatively easy approximations. CFE method is generally considered as worse of the two in the aspect of frequency response representation [6]. Oustaloup's method gives a very good representation of frequency response at selected band at the cost of high numerical sensitivity. This sensitivity can be lessened with use of time domain realizations [7, 8]. Different approach to approximation uses discrete realizations based on truncation of series representations. In particular, this class of methods includes truncation of Grünwald-Letnikov derivative definitions and power series expansions (PSE) in $z$ variable (discrete frequency) domain. These methods give good approximations only at high frequencies. Examples of the method can be found in [9]. Improvements on PSE methods with better band of correct approximation were investigated by Ferdi $[10,11]$. These two approaches, while being fundamentally different, have in common the fact that they are potentially effective but there are no proofs of their formal convergence to the actual noninteger order system. Another group of approximation methods uses diffusive realization of pseudodifferential operators [12] for approximation. First works in the area used finite-dimensional approximation with trapezoidal integration [13]. Later works used simple diagonal matrix time domain realization with modified Oustaloup nodes from frequency domain method [14-16]. This method is especially useful in analysis of 
infinite-dimensional systems using operator theory. It can be used, for example, to prove such properties as stability of closed-loop system [17].

The method of approximation analyzed in this paper is the Laguerre Impulse Response Approximation (LIRA) which is based on approximation of noninteger order system impulse response with Laguerre functions. Early works in this area were unsuccessful in approximation of $\alpha$ order integrators $[18,19]$. In [20], it was shown that under certain assumptions method is convergent in $\mathscr{L}_{1}$ and $\mathscr{L}_{2}$ norms when approximating asymptotically stable noninteger transfer functions of relative degree equal to or greater than one. Most applications of the method can be found in filtering and parametric optimization [21-23]. In [24], it was shown that in certain cases and at low orders of approximation LIRA outperforms Oustaloup method.

The main contribution of this paper is Theorem 6 which gives the justification of approximation of impulse responses that are in $\mathscr{L}_{1} \cap \mathscr{L}_{2}$. It was analyzed earlier in [20]; however, proof given in that paper was based on analysis of integrals and was not fully precise. Moreover, there was an assumption that impulse response has to be bounded which in the light of results of this paper is not necessary. In addition to that, Theorem 6 can be considered in spaces of multivariable functions. Additional contribution is Theorem 2 , which describes properties of functions in $\mathscr{L}_{1} \cap \mathscr{L}_{2}$. There is also series of corollaries and lemmas which allows explicit construction of approximation method. Methods' operation is illustrated with examples. The first example is qualitative where we explicitly verify the assumptions and other two come from applications: approximation of a filter and controller parameter optimization.

The rest of this paper is organized as follows. The first section contains the preliminaries necessary for theorems and lemmas presented further in the paper. In the main section, we prove the theorems concerning some properties of chosen types of functions and the main result concerning approximation in $\mathscr{L}_{1}$ space. Then explicit formulas for Laguerre Impulse Response Approximation method are given along with three examples: "fractional order lag" system, noninteger order filtering, and parametric optimization of noninteger order closed-loop system. The paper ends with conclusions and proposition of further extensions of the method.

\section{Preliminaries}

In this section, we present necessary definitions and notations that will be used throughout the paper.

We focus on stable noninteger order systems given by transfer functions like

$$
\begin{aligned}
\hat{g}(s) & =\frac{q_{m} s^{\gamma_{m}}+q_{m-1} s^{\gamma_{m-1}}+\cdots q_{0}}{s^{\sigma_{n}}+p_{n-1} s^{\sigma_{n-1}}+\cdots p_{0}} \\
\text { or } \hat{g}(s) & =\frac{1}{(T s+1)^{\alpha}}
\end{aligned}
$$

with zero initial conditions. Our interest is focused especially on their time domain representation in the form of convolution operator; that is,

$$
y(t)=u * g=\int_{0}^{t} u(t-\theta) g(\theta) d \theta .
$$

If realization in the form of noninteger differential equations is needed, we will, without the loss of generality, use Caputo definition of noninteger order derivative:

$$
{ }_{0}^{C} D_{t}^{\alpha} f(t)=\frac{1}{\Gamma(n-\alpha)} \int_{0}^{t} \frac{f^{(n)}(\tau) d \tau}{\left(t-\tau^{\alpha+1-n}\right)}
$$

where $\Gamma(\cdot)$ denotes the gamma function

$$
\Gamma(t)=\int_{0}^{\infty} x^{t-1} e^{-x} d x
$$

We will consider function spaces $\mathscr{L}_{1}(\Omega, \mathbb{R})$ and $\mathscr{L}_{2}(\Omega, \mathbb{R})$ of real functions $f: \Omega \rightarrow \mathbb{R}$, where $\Omega$ is an open subset of $\mathbb{R}^{m}$. Space $\mathscr{L}_{1}(\Omega, \mathbb{R})$ is a Banach space of absolute integrable functions with the norm

$$
\|f\|_{1}=\int_{\Omega}|f(\theta)| d \theta .
$$

Space $\mathscr{L}_{2}(\Omega, \mathbb{R})$ is a Hilbert space of square integrable functions, with norm

$$
\|f\|_{2}=\sqrt{\langle f, f\rangle}
$$

induced by the scalar product

$$
\sqrt{\langle f, g\rangle}=\int_{\Omega} f(\theta) g(\theta) d \theta
$$

Additionally, we will consider the Banach space $\mathscr{L}_{\infty}([0, \infty], \mathbb{R})$ of essentially bounded functions and a sequence space $\ell_{2}$ with norm

$$
\|c\|_{\ell_{2}}=\sqrt{\sum_{i} c_{i}^{2}}
$$

We will denote by $e_{i}, i=1,2, \ldots$, a set of orthonormal basis functions in $\mathscr{L}_{2}(\Omega, \mathbb{R})$. Additionally, for space $\mathscr{L}_{2}([0, \infty], \mathbb{R})$, we will consider orthonormal Laguerre functions denoted by $e_{i}(\theta, \mu)$ and given by

$$
e_{k}(\theta, \mu)=\sqrt{2 \mu} e^{-\mu \theta} l_{k}(2 \mu \theta), \quad k=0,1,2, \ldots,
$$

where $\mu$ is an arbitrary positive constant and $l_{k}$ is $k$ th Laguerre polynomial of the form

$$
l_{k}(z)=\frac{e^{z}}{k !} \frac{d^{k}}{d z^{k}}\left(e^{-z} z^{k}\right)
$$




\section{Main Results}

In this section, we will prove the theorem regarding $\mathscr{L}_{1}(\Omega, \mathbb{R})$ convergence of the function approximation and its consequences that can be used for approximation of convolution operator. In order to do so certain assumptions and lemmas have to be stated.

We will start with proving an important result regarding functions in $\mathscr{L}_{1}(\Omega, \mathbb{R}) \cap \mathscr{L}_{2}(\Omega, \mathbb{R})$.

Remark 1. In this paper, we will take the domain of a quotient of two functions $g$ and $h$ as a set, where $g / h$ exists.

Theorem 2. The following statements are equivalent:

(1) $f \in \mathscr{L}_{1}(\Omega, \mathbb{R}) \cap \mathscr{L}_{2}(\Omega, \mathbb{R})$.

(2) There exists a function $w \in \mathscr{L}_{2}(\Omega, \mathbb{R})$ such that $f / w \in$ $\mathscr{L}_{2}(\Omega, \mathbb{R})$.

Proof. (1) $\Rightarrow(2)$.

Let $v_{m}: \mathbb{R}^{m} \rightarrow \mathbb{R}$ be defined as

$$
v_{m}(\theta)=(1+|\theta|)^{m(1+\delta)}, \quad|\theta|=\sqrt{\theta^{\top} \theta},
$$

where $\delta>0$ is an arbitrary constant. Then $w=\sqrt{|f|}+v_{m}^{-1}$ is in $\mathscr{L}_{2}(\Omega, \mathbb{R})$. As $v_{m}(\theta)>0$, we have that $f / w \in \mathscr{L}_{2}(\Omega, \mathbb{R})$.

$(2) \Rightarrow(1)$.

We have

$$
\|f\|_{2}=\left\|\frac{f}{w} \cdot w\right\|_{2} \leq\|w\|_{2}\left\|\frac{f}{w}\right\|_{2}<\infty,
$$

so $f \in \mathscr{L}_{2}(\Omega, \mathbb{R})$. Using Hölder's inequality yields also the norm of $f$ in $\mathscr{L}_{1}(\Omega, \mathbb{R})$ space:

$$
\|f\|_{1}=\left\|\frac{f}{w} \cdot w\right\|_{1} \leq\|w\|_{2}\left\|\frac{f}{w}\right\|_{2}<\infty .
$$

Hence, $f \in \mathscr{L}_{1}(\Omega, \mathbb{R}) \cap \mathscr{L}_{2}(\Omega, \mathbb{R})$.

Remark 3. As an example of $f$ we can take bounded function in $\mathscr{L}_{1}(\Omega, \mathbb{R})$.

In order to prove the main result, we will need the following two lemmas.

Lemma 4. If there exists a function $w \in \mathscr{L}_{2}(\Omega, \mathbb{R})$, such that $f / w \in \mathscr{L}_{2}(\Omega, \mathbb{R})$ and $e_{i} / w \in \mathscr{L}_{2}(\Omega, \mathbb{R}), i=1,2, \ldots$, then

$$
\left\|\beta^{n}\right\|_{\ell_{2}}<\infty
$$

where

$$
\beta_{j}^{n}=\sum_{i=0}^{n} \alpha_{i} \gamma_{j}^{i}
$$

and $\gamma_{j}^{i}=\left\langle e_{i} / w, e_{j}\right\rangle=\gamma_{i}^{j}$ and $\alpha_{i}=\left\langle f, e_{i}\right\rangle$.

Proof. As $e_{i} / w \in \mathscr{L}_{2}(\Omega, \mathbb{R})$, we can write function $e_{i} / w$ as a series expansion of orthonormal basis functions in $\mathscr{L}_{2}(\Omega, \mathbb{R})$ :

$$
\frac{e_{i}}{w}=\sum_{j=0}^{\infty} \gamma_{j}^{i} e_{j}
$$

with coefficients $\gamma_{j}^{i}=\left\langle e_{i} / w, e_{j}\right\rangle=\gamma_{i}^{j}$. Therefore, we have

$$
e_{i}=w \sum_{j=0}^{\infty} \gamma_{j}^{i} e_{j}
$$

The same operation is done for $f$ and $f / w$, since both are in $\mathscr{L}_{2}(\Omega, \mathbb{R})$ as proven in Theorem 2 . We have

$$
f=\sum_{i=0}^{\infty} \alpha_{i} e_{i}
$$

where $\alpha_{i}=\left\langle f, e_{i}\right\rangle$ and

$$
\frac{f}{w}=\sum_{j=0}^{\infty} \beta_{j} e_{j}
$$

where $\beta_{j}=\left\langle f / w, e_{j}\right\rangle$. On the other hand, we have

$$
\frac{f}{w}=\frac{1}{w} \sum_{i=0}^{\infty} \alpha_{i} e_{i}
$$

Let us take partial sum of series (20). As it is convergent in $\mathscr{L}_{2}(\Omega, \mathbb{R})$, also norm of its partial sum is finite. We have then

$$
\begin{aligned}
\frac{1}{w} \sum_{i=0}^{n} \alpha_{i} e_{i} & =\frac{1}{(17)} \sum_{i=0}^{n}\left(\alpha_{i} \cdot\left(w \cdot \sum_{j=0}^{\infty} \gamma_{j}^{i} e_{j}\right)\right) \\
& =\sum_{i=0}^{n} \sum_{j=0}^{\infty} \alpha_{i} \gamma_{j}^{i} e_{j}=\sum_{j=0}^{\infty} e_{j} \sum_{i=0}^{n} \alpha_{i} \gamma_{j}^{i} \underset{(15)}{=} \sum_{j=0}^{\infty} \beta_{j}^{n} e_{j} .
\end{aligned}
$$

As norm in $\mathscr{L}_{2}(\Omega, \mathbb{R})$ of left side of $(21)$ is finite, we have

$$
\left\|\sum_{j=0}^{\infty} \beta_{j}^{n} e_{j}\right\|=\sum_{j=0}^{\infty}\left(\beta_{j}^{n}\right)^{2}=\left\|\beta^{n}\right\|_{\ell_{2}}<\infty
$$

which implies thesis.

Lemma 5. Let $c^{n}=\left(c_{j}^{n}\right), n, j=0,1,2, \ldots$, be a family of sequences parametrized by $n$, such that

(1) $\forall j:\left|c_{j}^{n}\right| \rightarrow 0$ when $n \rightarrow \infty$,

(2) $\forall n:\left\|c^{n}\right\|_{\ell_{2}}<\infty$.

Then

$$
\left\|c^{n}\right\|_{\ell_{2}} \longrightarrow 0 \quad \text { when } n \longrightarrow \infty \text {. }
$$

Proof. Let us consider $\varepsilon>0$. The sequence $\left(c_{j}^{n}\right)$ is squaresummable with respect to $j$ for all $n$. Therefore, we can find a function $\mathbb{R}^{+} \rightarrow \mathbb{N}: m(\varepsilon)$ such that

$$
\sum_{j=m(\varepsilon)+1}^{\infty}\left|c_{j}^{0}\right|^{2}<\frac{1}{2} \varepsilon
$$


Using the first assumption, we have

$$
\begin{aligned}
\sum_{j=0}^{\infty}\left|c_{j}^{n}\right|^{2} & =\sum_{j=0}^{m(\varepsilon)}\left|c_{j}^{n}\right|^{2}+\sum_{j=m(\varepsilon)+1}^{\infty}\left|c_{j}^{n}\right|^{2} \\
& \leq \sum_{j=0}^{m(\varepsilon)}\left|c_{j}^{n}\right|^{2}+\sum_{j=m(\varepsilon)+1}^{\infty}\left|c_{j}^{0}\right|^{2} \leq \sum_{j=0}^{m(\varepsilon)}\left|c_{j}^{n}\right|^{2}+\frac{1}{2} \varepsilon .
\end{aligned}
$$

From assumption (1), we know that we can choose $n_{0}$, such that $\left|c_{j}^{n_{0}}\right| \leq \varepsilon / 2 m(\varepsilon)$, for all $j=0,1,2, \ldots, m(\varepsilon)$ and that

$$
\sum_{j=0}^{\infty}\left|c_{j}^{n}\right|^{2}<\varepsilon \quad \forall n \geq n_{0} .
$$

Therefore, for any $\varepsilon>0$, there is $n_{0}$, such that $\left\|c^{n}\right\|_{\ell_{2}}<\varepsilon$ for all $n \geq n_{0}$.

Now we can formulate and prove the intended theorem.

Theorem 6. If there exists a function $w \in \mathscr{L}_{2}(\Omega, \mathbb{R})$, such that $f / w \in \mathscr{L}_{2}(\Omega, \mathbb{R})$ and $e_{i} / w \in \mathscr{L}_{2}(\Omega, \mathbb{R})$, where $e_{i}$ are functions of orthonormal basis in $\mathscr{L}_{2}(\Omega, \mathbb{R}) i=1,2, \ldots$, then

$$
\left\|\sum_{i=0}^{n}\left\langle f, e_{i}\right\rangle e_{i}-f\right\|_{1} \longrightarrow 0 \quad \text { when } n \longrightarrow \infty .
$$

Proof. From Theorem 2 and assumptions, we know that $f$, $f / w$, and $e_{i} / w$ are in $\mathscr{L}_{2}(\Omega, \mathbb{R})$ and as such may be written in form of infinite series:

$$
\begin{aligned}
& e_{i}=w \sum_{j=0}^{\infty} \gamma_{j}^{i} e_{j}, \\
& f=\sum_{i=0}^{\infty} \alpha_{i} e_{i}, \\
& \frac{f}{w}=\sum_{j=0}^{\infty} \beta_{j} e_{j}=\frac{1}{w} \sum_{i=0}^{\infty} \alpha_{i} e_{i},
\end{aligned}
$$

with coefficients $\gamma_{j}^{i}=\left\langle e_{i} / w, e_{j}\right\rangle=\gamma_{i}^{j}, \alpha_{i}=\left\langle f, e_{i}\right\rangle$, and $\beta_{j}=$ $\left\langle f / w, e_{j}\right\rangle$, respectively. Let

$$
\beta_{j}^{n}=\sum_{i=0}^{n} \alpha_{i} \gamma_{j}^{i}
$$

The following estimate holds:

$$
\begin{gathered}
\left\|\sum_{i=0}^{n} \alpha_{i} e_{i}-f\right\|_{1} \underset{(28)}{=}\left\|\sum_{i=0}^{n} \alpha_{i}\left(w \sum_{j=0}^{\infty} \gamma_{j}^{i} e_{j}\right)-\frac{f}{w} w\right\|_{1} \\
=\left\|w\left(\sum_{j=0}^{\infty} \beta_{j}^{n} e_{j}-\frac{f}{w}\right)\right\|_{1} \\
\leq \quad\|w\|_{2}\left\|\sum_{j=0}^{\infty} \beta_{j}^{n} e_{j}-\frac{f}{w}\right\|_{2} \\
\text { Hölder } \\
=\|w\|_{2}\left\|\sum_{j=0}^{\infty}\left(\beta_{j}^{n}-\beta_{j}\right) e_{j}\right\|_{2} .
\end{gathered}
$$

Let us denote $c^{n}$ as a sequence with elements $c_{j}^{n}=\beta_{j}-\beta_{j}^{n}$. From (32) and orthonormality of the basis $e_{i}$, we have

$$
\left\|\sum_{i=0}^{n} \alpha_{i} e_{i}-f\right\|_{1} \leq\|w\|_{2}\left\|c^{n}\right\|_{\ell_{2}} .
$$

We will investigate the behavior of the sequence $c^{n}$. Using linearity of inner product in $\mathscr{L}_{2}(\Omega, \mathbb{R})$, we have

$$
\begin{aligned}
\beta_{j} & =\left\langle\frac{f}{w}, e_{j}\right\rangle=\underset{(28)}{=}\left\langle\frac{f}{w}, w \sum_{i=0}^{\infty} \gamma_{i}^{j} e_{i}\right\rangle \\
& =\sum_{i=0}^{\infty}\left\langle\frac{f}{w} w, e_{i}\right\rangle \gamma_{i}^{j}=\sum_{i=0}^{\infty} \alpha_{i} \gamma_{i}^{j}=\beta_{j}^{n}+\sum_{i=n+1}^{\infty} \alpha_{i} \gamma_{j}^{i} ;
\end{aligned}
$$

hence,

$$
\beta_{j}-\beta_{j}^{n}=c_{j}^{n}=\sum_{i=n+1}^{\infty} \alpha_{i} \gamma_{j}^{i} .
$$

Using the Schwartz inequality, we have

$$
\left|c_{j}^{n}\right| \underset{(34)}{\leq} \sum_{i=n+1}^{\infty}\left|\alpha_{i} \gamma_{i}^{j}\right|_{\text {Schwartz }}^{\leq} \sqrt{\sum_{i=n+1}^{\infty}\left|\alpha_{i}\right|^{2}} \sqrt{\sum_{i=n+1}^{\infty}\left|\gamma_{j}^{i}\right|^{2}} .
$$

$f \in \mathscr{L}_{2}(\Omega, \mathbb{R})$ and (36) imply that $\left|c_{j}^{n}\right| \rightarrow 0$ when $n \rightarrow \infty$, for all $j=0,1,2, \ldots$. From Lemma 4 sequence $\left(\beta_{j}^{n}\right)$ is squaresummable for all $n$. From properties of series expansion in $\mathscr{L}_{2}(\Omega, \mathbb{R})$, we can conclude that $\beta_{j}$ is also square-summable. As a consequence, the sequence $c^{n}$ is square-summable. The assertion of the theorem follows from inequality (33) and Lemma 5.

Remark 7. Although Theorem 2 guarantees the existence of $w$ for every function $f$ in $\mathscr{L}_{1}(\Omega, \mathbb{R}) \cap \mathscr{L}_{2}(\Omega, \mathbb{R})$, it does not ensure that $e_{i} / w \in \mathscr{L}_{2}(\Omega, \mathbb{R})$ for a given basis. Therefore, the choice of appropriate basis functions determines the existence of approximation.

As a direct consequence of Theorem 6, we have the following results.

Corollary 8. If $f \in \mathscr{L}_{1}\left(\mathbb{R}^{m}, \mathbb{R}\right) \cap \mathscr{L}_{2}\left(\mathbb{R}^{m}, \mathbb{R}\right)$ and there exists a basis $e_{i} \in \mathscr{L}_{2}\left(\mathbb{R}^{m}, \mathbb{R}\right)$, such that $e_{i} v_{m} \in \mathscr{L}_{2}\left(\mathbb{R}^{n}, \mathbb{R}\right)$ for $i=$ $0,1,2, \ldots$, with $v_{m}$ given by (11), then

$$
\left\|\sum_{i=0}^{n} \alpha_{i} e_{i}-f\right\|_{1} \longrightarrow 0 \quad \text { when } n \longrightarrow \infty .
$$

Proof. From Theorem 2, we know that there exists $w$ such that $f / w \in \mathscr{L}_{2}\left(\mathbb{R}^{m}, \mathbb{R}\right)$. Using the assumption that $e_{i} v_{m} \in$ $\mathscr{L}_{2}\left(\mathbb{R}^{m}, \mathbb{R}\right)$, we have $e_{i} / w \in \mathscr{L}_{2}\left(\mathbb{R}^{m}, \mathbb{R}\right)$. So the assumptions of Theorem 6 are fulfilled which completes the proof.

Corollary 9 (convergence of Laguerre series). If $f \in$ $\mathscr{L}_{1}([0, \infty], \mathbb{R}) \cap \mathscr{L}_{2}([0, \infty], \mathbb{R})$ and $e_{i}(\mu, \theta)$ are orthonormal Laguerre functions, then

$$
\left\|\sum_{i=0}^{n} \alpha_{i} e_{i}-f\right\|_{1} \longrightarrow 0 \quad \text { when } n \longrightarrow \infty
$$


where

$$
\alpha_{i}=\int_{0}^{\infty} e_{i}(\mu, \theta) f(\theta) d \theta
$$

Proof. Because for $v_{1}$ from Corollary 8 we have $e_{i} v_{1} \in$ $\mathscr{L}_{2}([0, \infty], \mathbb{R})$ for $i=0,1,2, \ldots$, the assumptions of Corollary 8 are fulfilled.

Corollary 10 (convolution approximation in Laguerre basis). Let $f \in \mathscr{L}_{1}([0, \infty], \mathbb{R}) \cap \mathscr{L}_{2}([0, \infty], \mathbb{R})$ and $u \in$ $\mathscr{L}_{\infty}([0, \infty], \mathbb{R})$, then for

$$
\begin{gathered}
y(t)=\int_{0}^{t} u(t-\theta) f(\theta) d \theta, \\
y_{n}(t)=\int_{0}^{t} u(t-\theta) f_{n}(\theta) d \theta,
\end{gathered}
$$

where

$$
f_{n}(\theta)=\sum_{i=0}^{n} \alpha_{i} e_{i}(\theta, \mu)
$$

with $\alpha_{i}$ given by (39), we have

$$
\left|y(t)-y_{n}(t)\right| \longrightarrow 0 \quad \text { when } n \longrightarrow \infty, \forall t \geq 0 .
$$

Proof. Using Hölder's inequality, we have

$$
\left|y(t)-y_{n}(t)\right| \leq\|u\|_{\infty}\left\|\sum_{i=0}^{n} \alpha_{i} e_{i}(\mu, \theta)-f\right\|_{1} .
$$

Applying Corollary 9 ends the proof.

\section{Laguerre Impulse Response Approximation Method}

Corollaries 9 and 10 give theoretical basis for approximating convolution operator (2) with a Laguerre series. In the following we present three lemmas that allow practical construction of approximant for systems described with SISO (single-input single-output) noninteger transfer functions.

Lemma 11 (computation of scalar products). If $g(t) \in$ $\mathscr{L}_{2}([0, \infty], \mathbb{R})$ has a Laplace transform $\hat{g}(s)$, then

$$
\left\langle g(\theta), e_{k}(\theta, \mu)\right\rangle=\frac{\sqrt{2 \mu}}{k !} \sum_{j=0}^{k}\left(\begin{array}{l}
k \\
j
\end{array}\right) c_{j}^{k}(\mu) \hat{g}^{(k-j)}(\mu),
$$

where $e_{i}(\theta, \mu)$ is the ith orthonormal Laguerre function with parameter $\mu$ :

$$
\begin{aligned}
c_{j}^{k} & =\frac{k-j+1}{2 \mu} c_{j-1}^{k}, \\
c_{0}^{k}(\mu) & =(2 \mu)^{k}, \quad j=1,2, \ldots, k, \\
\hat{g}^{(j)}(s) & =\frac{d^{j} \hat{g}(s)}{d s^{j}} .
\end{aligned}
$$

Proof. Proof is given in [25, page 65].

Lemma 12 (choice of optimal $\mu)$. Let $g(\theta) \in \mathscr{L}_{2}([0, \infty], \mathbb{R})$ and

$$
g_{n}(\theta)=\sum_{i=0}^{n} \beta_{i}(\mu) e_{i}(\theta, \mu),
$$

where $\beta_{i}(\mu)=\left\langle g(\theta), e_{i}(\theta, \mu)\right\rangle$ and $e_{i}(\theta, \mu)$ is the ith orthonormal Laguerre function with parameter $\mu$. The norm of the difference $\left\|g-g_{n}\right\|_{2}$ for given $n$ is minimal if

$$
\mu=\arg \max _{\mu} \sum_{k=0}^{n} \beta_{k}^{2}(\mu) .
$$

Proof. We will compute the square of error norm (arguments are dropped):

$$
\begin{aligned}
\left\|g-g_{n}\right\|_{2}^{2} & =\int_{0}^{\infty}\left(g-g_{n}\right)^{2} d t \\
& =\int_{0}^{\infty} g^{2} d t-2 \int_{0}^{\infty} g g_{n} d t+\int_{0}^{\infty} g_{n}^{2} d t \\
& =\|g\|_{2}^{2}+\sum_{k=0}^{n} \beta_{k}^{2}(\mu)-2 \int_{0}^{\infty} g \sum_{k=0}^{n} \beta_{k}(\mu) e_{k} d t \\
& =\|g\|_{2}^{2}+\sum_{k=0}^{n} \beta_{k}^{2}(\mu)-2 \sum_{k=0}^{n} \beta_{k}^{2}(\mu) \\
& =\|g\|_{2}^{2}-\sum_{k=0}^{n} \beta_{k}^{2}(\mu) .
\end{aligned}
$$

It can be easily seen that the above expression is minimal when thesis is fulfilled.

Lemma 13 (convolution state space realization). The convolution of $u \in \mathscr{L}_{\infty}([0, \infty], \mathbb{R})$ with $g_{n}$ given by

$$
y_{n}(t)=\int_{0}^{t} u(t-\theta) g_{n}(\theta) d \theta,
$$

where

$$
g_{n}(\theta)=\sum_{i=0}^{n} \beta_{i} e_{i}(\theta, \mu)
$$

where $e_{i}(\theta, \mu)$ is the ith orthonormal Laguerre function with parameter $\mu$, is equal to the solution of the following system of differential equations:

$$
\begin{aligned}
& \dot{\xi}=\left[\begin{array}{cccccc}
-\mu & 0 & 0 & 0 & \cdots & 0 \\
-2 \mu & -\mu & 0 & 0 & \cdots & 0 \\
-2 \mu & -2 \mu & -\mu & 0 & \cdots & 0 \\
-2 \mu & -2 \mu & -2 \mu & -\mu & \cdots & 0 \\
\vdots & \vdots & \vdots & \vdots & \ddots & \vdots \\
-2 \mu & -2 \mu & -2 \mu & \cdots & -2 \mu & -\mu
\end{array}\right] \boldsymbol{\xi}+\left[\begin{array}{c}
\sqrt{2 \mu} \\
\sqrt{2 \mu} \\
\sqrt{2 \mu} \\
\sqrt{2 \mu} \\
\vdots \\
\sqrt{2 \mu}
\end{array}\right] u \\
& y_{n}=\left[\begin{array}{lllllll}
\beta_{0} & \beta_{1} & \beta_{2} & \beta_{3} & \beta_{4} & \cdots & \beta_{n}
\end{array}\right] \xi
\end{aligned}
$$

with $\boldsymbol{\xi}(0)=0$. 
Proof. It can be easily seen that

$$
y(t)=\sum_{k=0}^{n} \beta_{k} \int_{0}^{t} e_{k}(t-\theta, \mu) u(\theta) d \theta .
$$

Let us set

$$
\xi_{k}(t)=\int_{0}^{t} e_{k}(t-\theta, \mu) u(\theta) d \theta
$$

which along with (53) gives (52). From boundedness of $u$ and definition (54), we get $\xi_{k}(0)=0$.

We will now use two additional facts. First, the derivative of a Laguerre function with respect to first argument can be expressed with Laguerre functions of the same and lower order; that is,

$$
\dot{e}_{k}(\theta, \mu)=-\mu e_{k}(\theta, \mu)-2 \mu \sum_{i=0}^{k-1} e_{i}(\theta, \mu)
$$

Additionally, the derivative of the convolution operator with respect to $t$ is given by

$$
\begin{aligned}
& \frac{d}{d t} \int_{0}^{t} f(t-\tau) g(\tau) d \tau \\
& \quad=f(0) g(t)+\int_{0}^{t} \dot{f}(t-\tau) g(\tau) d \tau
\end{aligned}
$$

Differentiating (54) and using (55) and (56), we obtain

$$
\dot{\xi}_{k}=-\mu \xi_{k}-2 \mu \sum_{i=0}^{k-1} \xi_{i}+\sqrt{2 \mu} u
$$

Collecting (57) for $k=0,1, \ldots, n$ leads to (51).

Lemma 11 enables efficient computation of approximation coefficients. Lemma 12 shows how to choose the basis parameter $\mu$ allowing minimal approximation error for given approximation order. Finally, Lemma 13 gives a tool how to construct a state space (or if needed transfer function) realization of the approximant allowing among the others simulation, filtering, use in real time applications, or optimization.

We will now present three examples of using the proposed method for approximation of noninteger order systems.

4.1. "Fractional Order Lag". This class of systems is widely used in various applications, usually without serious analysis. These systems are classified as noninteger order; however, their realization is in the form of noninteger differential equations (at least not their finite number). Their transfer function takes the form

$$
\widehat{g}(s)=\frac{1}{(T s+1)^{\alpha}},
$$

where $T$ is a constant. Impulse response for (58) can be found using inverse Laplace transform:

$$
\begin{aligned}
g(t) & =\mathscr{L}^{-1}\left\{\frac{1}{(T s+1)^{\alpha}}\right\} \\
& =\mathscr{L}^{-1}\left\{\frac{1}{T^{\alpha}(s+1 / T)^{\alpha}}\right\} \\
& =\frac{1}{T^{\alpha}} \cdot \mathscr{L}^{-1}\left\{\frac{1}{(s+1 / T)^{\alpha}}\right\} \\
& =\frac{1}{T^{\alpha}} \cdot e^{-1 / T} \mathscr{L}^{-1}\left\{\frac{1}{s^{\alpha}}\right\} \\
& =\frac{1}{T^{\alpha}} \cdot \frac{t^{\alpha-1} e^{-t / T}}{\Gamma(\alpha)},
\end{aligned}
$$

where $\Gamma(x)$ denotes gamma function [26]. It can be easily verified that (63) for $\alpha \geq 1$ is bounded and its $\mathscr{L}_{1}([0, \infty], \mathbb{R})$ norm can be easily computed. The impulse response is positive for all $t \geq 0$; therefore, the absolute value in norm can be omitted. Consider

$$
\int_{0}^{t}|g(t)| d t=\int_{0}^{t} g(t) d t=h(t) .
$$

Therefore

$$
\int_{0}^{\infty}|g(t)| d t=\lim _{t \rightarrow \infty} h(t)-\lim _{t \rightarrow 0} h(t)
$$

and it is sufficient to use initial and final value theorems:

$$
\begin{aligned}
& \lim _{t \rightarrow \infty} h(t)=\lim _{s \rightarrow 0}\left(s \cdot \frac{1}{s(T s+1)^{\alpha}}\right)=1, \\
& \lim _{t \rightarrow 0} h(t)=\lim _{s \rightarrow \infty}\left(s \cdot \frac{1}{s(T s+1)^{\alpha}}\right)=0 .
\end{aligned}
$$

Because of that and Remark 3 , we can see that $g \in$ $\mathscr{L}_{1}([0, \infty], \mathbb{R}) \cap \mathscr{L}_{2}([0, \infty], \mathbb{R})$.

However, it can be shown that while for $\alpha \in(1 / 2,1)$ the impulse response is unbounded the assumptions of Theorem 6 are fulfilled.

Corollary 14. Approximation of function

$$
g(t)=\frac{1}{T^{\alpha}} \cdot \frac{t^{\alpha-1} e^{-t / T}}{\Gamma(\alpha)}
$$

with Laguerre series is convergent in the sense of $\mathscr{L}_{1}(\Omega, \mathbb{R})$ norm.

Proof. We will prove that function (67) fulfills the assumptions of Theorem 6.

First we will prove that $g \in \mathscr{L}_{2}(\Omega, \mathbb{R})$. The following calculations are valid:

$$
\begin{aligned}
\int_{0}^{\infty} g^{2}(t) d t & =\frac{1}{T^{2 \alpha} \cdot \Gamma^{2}(\alpha)} \int_{0}^{\infty} t^{2 \alpha-2} e^{-2 t / T} d t \\
& =-\left.\frac{2^{1-2 \alpha} T^{2 \alpha-1}}{T^{2 \alpha} \cdot \Gamma^{2}(\alpha)} \cdot \Gamma\left(2 \alpha-1, \frac{2 t}{T}\right)\right|_{0} ^{\infty},
\end{aligned}
$$


where

$$
\Gamma(a, x)=\int_{x}^{\infty} t^{a-1} e^{-t} d t
$$

is the "upper" incomplete gamma function and $\operatorname{Re} a>0$.

The limit of (68) for $t \rightarrow 0$ can be written as

$$
\begin{aligned}
\lim _{t \rightarrow 0} & \left(-\frac{2^{1-2 \alpha} T^{\alpha-1}}{\Gamma(\alpha)} \cdot \Gamma\left(2 \alpha-1, \frac{2 t}{T}\right)\right) \\
& =-\frac{2^{1-2 \alpha} T^{\alpha-1}}{\Gamma(\alpha)} \lim _{t \rightarrow 0} \Gamma\left(2 \alpha-1, \frac{2 t}{T}\right) \\
& =-\frac{2^{1-2 \alpha} T^{\alpha-1}}{\Gamma(\alpha)} \Gamma(2 \alpha-1,0) \\
& =-\frac{2^{1-2 \alpha} T^{\alpha-1}}{\Gamma(\alpha)} \Gamma(2 \alpha-1)
\end{aligned}
$$

and is bounded for $2 \alpha-1>0$; that is, $\alpha>1 / 2$. The same analysis was conducted for the second limit $t \rightarrow \infty$ :

$$
\begin{gathered}
\lim _{t \rightarrow \infty}\left(-\frac{2^{1-2 \alpha} T^{\alpha-1}}{\Gamma(\alpha)} \cdot \Gamma\left(2 \alpha-1, \frac{2 t}{T}\right)\right)=-\frac{2^{1-2 \alpha} T^{\alpha-1}}{\Gamma(\alpha)} \\
\cdot \lim _{t \rightarrow \infty} \Gamma\left(2 \alpha-1, \frac{2 t}{T}\right)=-\frac{2^{1-2 \alpha} T^{\alpha-1}}{\Gamma(\alpha)} \\
. \lim _{t \rightarrow \infty}\left(\int_{0}^{\infty} x^{2 \alpha-2} e^{-x} d x-\int_{0}^{2 t / T} x^{2 \alpha-2} e^{-x} d x\right)=0 .
\end{gathered}
$$

From formulas (70) and (71), one obtains that the $\mathscr{L}_{2}(\Omega, \mathbb{R})$ norm of $g(t)$ is bounded for $\alpha \in(1 / 2,1)$.

Next, it can be easily seen that setting

$$
w=e^{-\varepsilon t}
$$

for $\varepsilon>0$ gives $f / w \in \mathscr{L}_{2}(\Omega, \mathbb{R})$ and $e_{i}(\mu, \theta) / w \in \mathscr{L}_{2}(\Omega, \mathbb{R})$, for $i=0,1,2$ and $\mu>\varepsilon$, which completes the proof.

We will now present examples of approximation for different "fractional order lags," one for each case. In this example and in the next example, we will analyze three types of plots. We will compare impulse responses of original noninteger order system with one of its approximants (with $\mu$ chosen according to Lemma 12) and with an approximant with nonoptimal $\mu$. Analogous comparison will be performed with bode plots, which are a good method for transfer function evaluation. Finally, we will present the function $\sum_{k=0}^{n} \beta_{k}^{2}(\mu)$ with marked optimal and nonoptimal values of $\mu$. In case of fractional order lag approximations presented are of order 20 .

In Figures 1(a), 2(a), and 3(a), we analyze fractional order lag with $\alpha=0.7$ and $T=1$. As it can be seen in Figure 1(a), while both approximants are bounded, they rather closely approximate the unbounded impulse response. Differences with optimal and nonoptimal $\mu$ are especially visible in Figure 2(a), where magnitude and phase start to diverge for higher frequencies. It should be noted that phase approximation is strongly oscillatory. As it can be seen in Figure 3(a), function maximized with respect to $\mu$ is unimodal; however, the more complicated system becomes the function changes.

In Figures 1(b), 2(b), and 3(b), we can see analogous analysis for fractional order lag with $\alpha=1.5$ and $T=1$. In Figure 1(b), the differences between optimal and nonoptimal $\mu$ are more visible. Frequency responses for optimal $\mu$ are also better and less oscillatory (see Figure 2(b)). Finally, the analysis of $\mu$ optimization shows much more irregular shape of maximized function.

More detailed analysis of Laguerre Impulse Response Approximation method for fractional order lag can be found in [23].

4.2. Noninteger Order Filter. The main area of application of LIRA method investigated by authors was the realization of noninteger order filters. Our focus was mostly on generalizations of second-order filters developed by Radwan et al. [27]. Detailed analysis of method operation was presented in [24]. Here we will present only a brief description of the idea, with an example of low-pass filter. We consider a filter in a form

$$
G(s)=\frac{1}{s^{2 \alpha}+b s^{\alpha}+c},
$$

where $a$ and $b$ fulfill the conditions of stability (see $[28,29])$.

Remark 15. We do not have at the moment condition when the noninteger transfer function has an impulse response in $\mathscr{L}_{2}([0, \infty], \mathbb{R})$. At the moment working hypothesis is that sufficient conditions are stability and transfer function's relative degree (difference between max. degree of denominator and max. degree of numerator) is at least 1. Despite multiple trials, no counter example was found.

Equivalent representation of (73) is the realization in the form of a system of differential equations of order $\alpha$. This system can take the form (see [30])

$$
\begin{aligned}
{ }_{0}^{C} D_{t}^{\alpha} \mathbf{x}(t) & =\mathbf{A} \mathbf{x}(t)+\mathbf{B} u(t), \\
y(t) & =\mathbf{C} \mathbf{x}(t)
\end{aligned}
$$

with the following matrices:

$$
\begin{aligned}
& \mathbf{A}=\left[\begin{array}{cc}
0 & 1 \\
-b & -a
\end{array}\right], \\
& \mathbf{B}=\left[\begin{array}{l}
0 \\
1
\end{array}\right], \\
& \mathbf{C}=\left[\begin{array}{ll}
1 & 0
\end{array}\right] .
\end{aligned}
$$

The impulse response of filter (73) is given by [30]

$$
g(t)=t^{\alpha-1} \mathbf{C E}_{\alpha, \alpha}\left(\mathbf{A} t^{\alpha}\right) \mathbf{B},
$$

where $\mathbf{E}_{\alpha, \alpha}(x)$ is the two-parameter Mittag-Leffler function.

In order to illustrate the operation of LIRA for filter we have chosen one, for which poles are a conjugate pair in right 


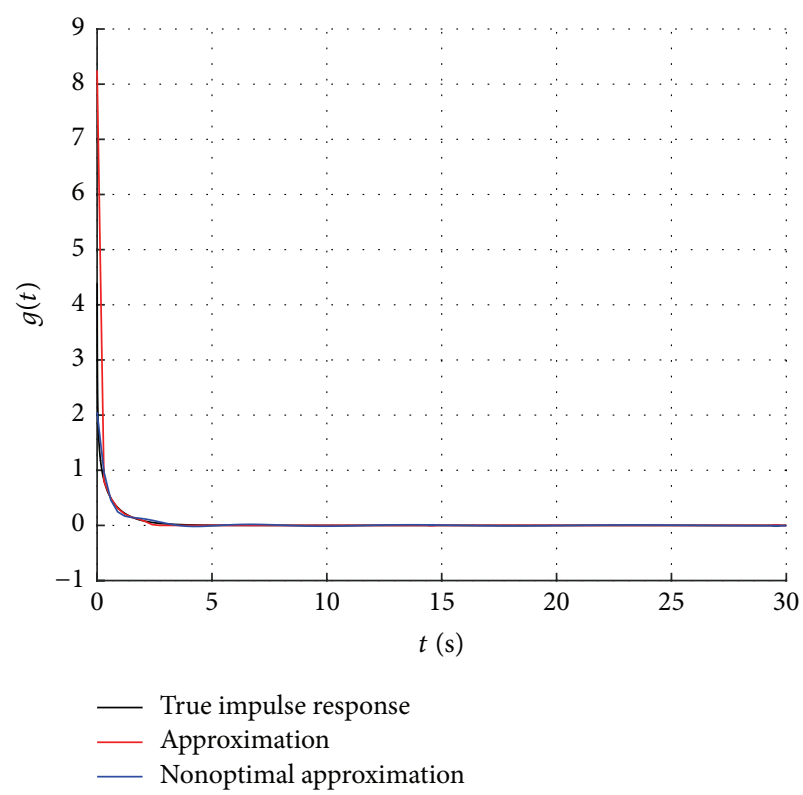

(a) Impulse response for $\alpha=0.7$

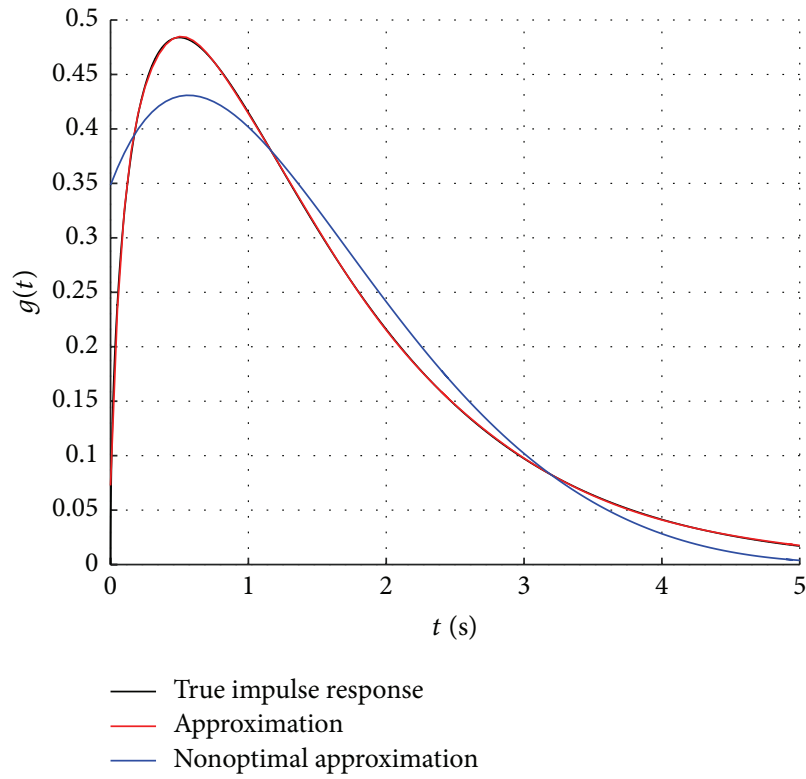

(b) Impulse response for $\alpha=1.5$

FIgURE 1: Impulse responses for two values of $\alpha$.
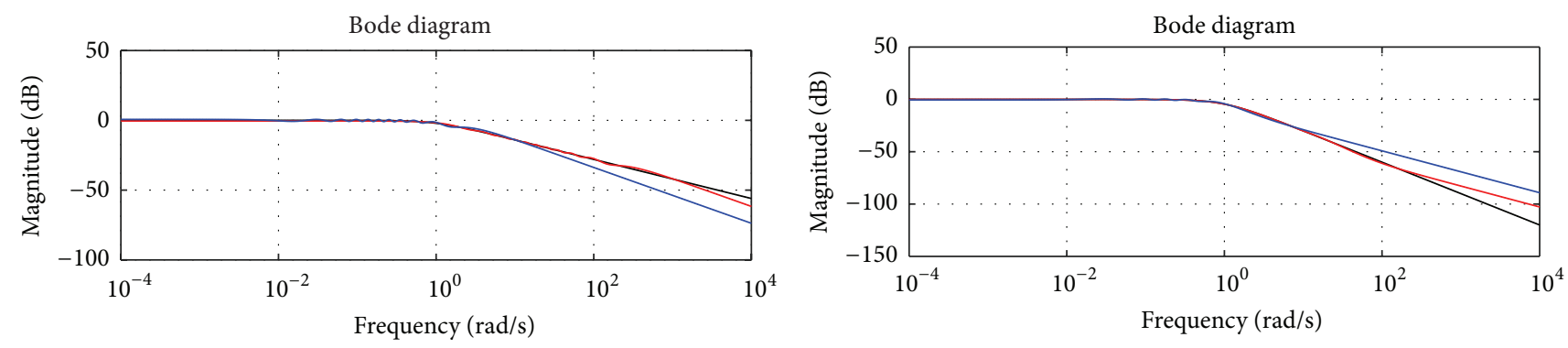

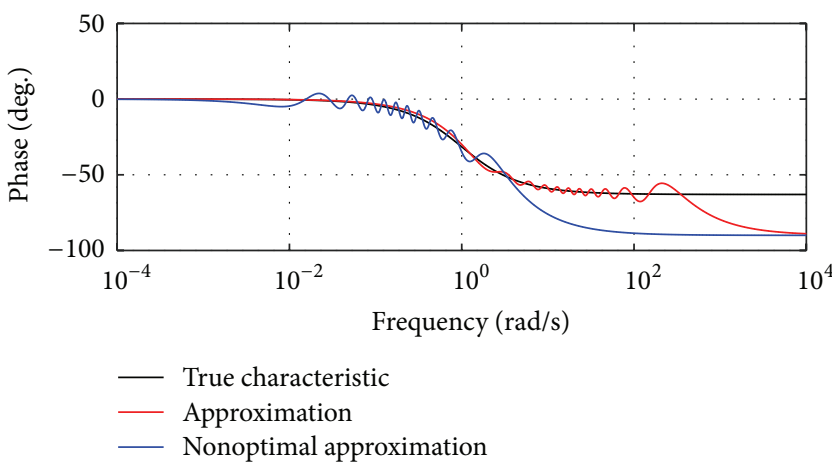

(a) Frequency response for $\alpha=0.7$

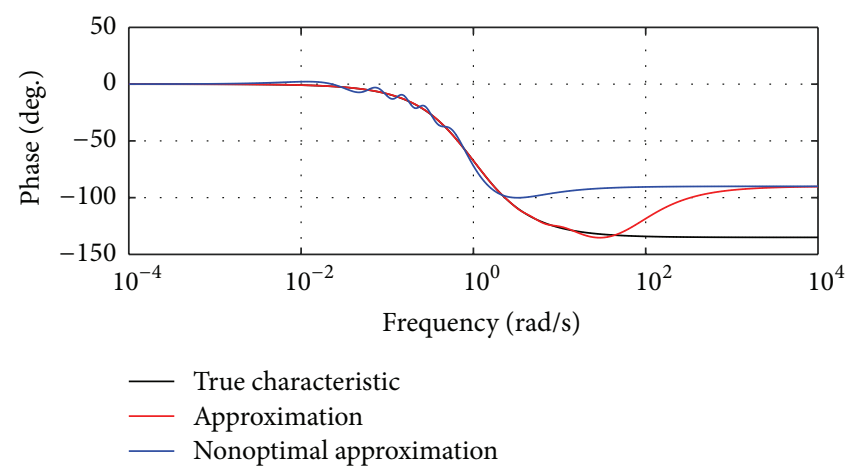

(b) Impulse response for $\alpha=1.5$

Figure 2: Frequency responses for two values of $\alpha$.

complex half plane. Such filters are oscillatory and always have resonance peak. We compare both impulse responses and frequency responses. All approximations are of 25th order with optimally and nonoptimally chosen $\mu$. The case is presented in Figure 4. As it can be seen in Figure 4(a), the optimal approximant of impulse response is almost indistinguishable from the exact value. Nonoptimal one well approximates initial behavior but for longer time stops at zero. In the frequency response (Figure 4(b)) the optimal approximation is again almost indistinguishable from noninteger order filter. However, nonoptimal $\mu$ causes oscillatory behavior on magnitude and phase responses. Moreover, 


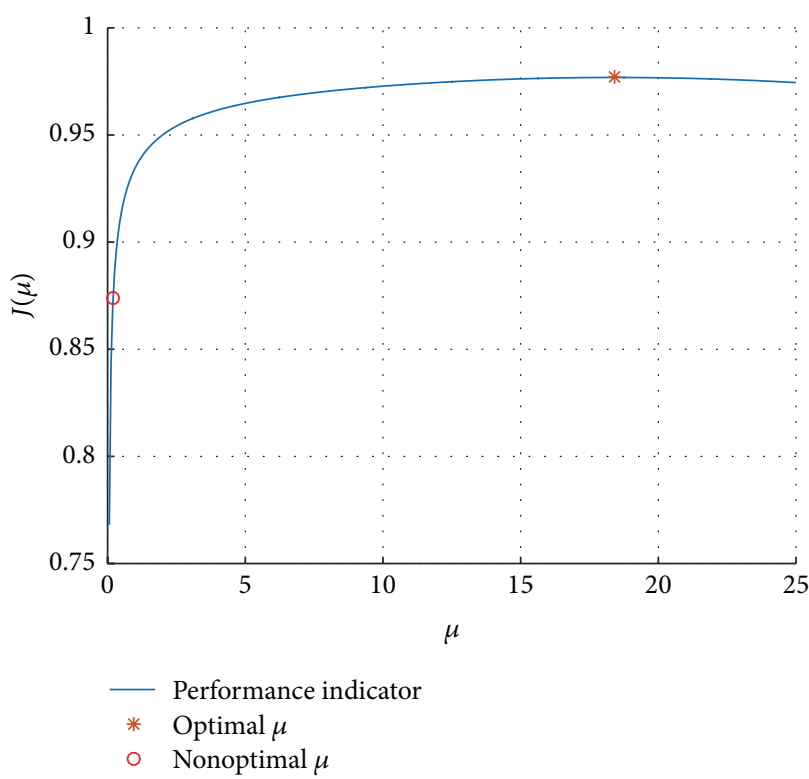

(a) Analysis of $\mu$ for $\alpha=0.7$

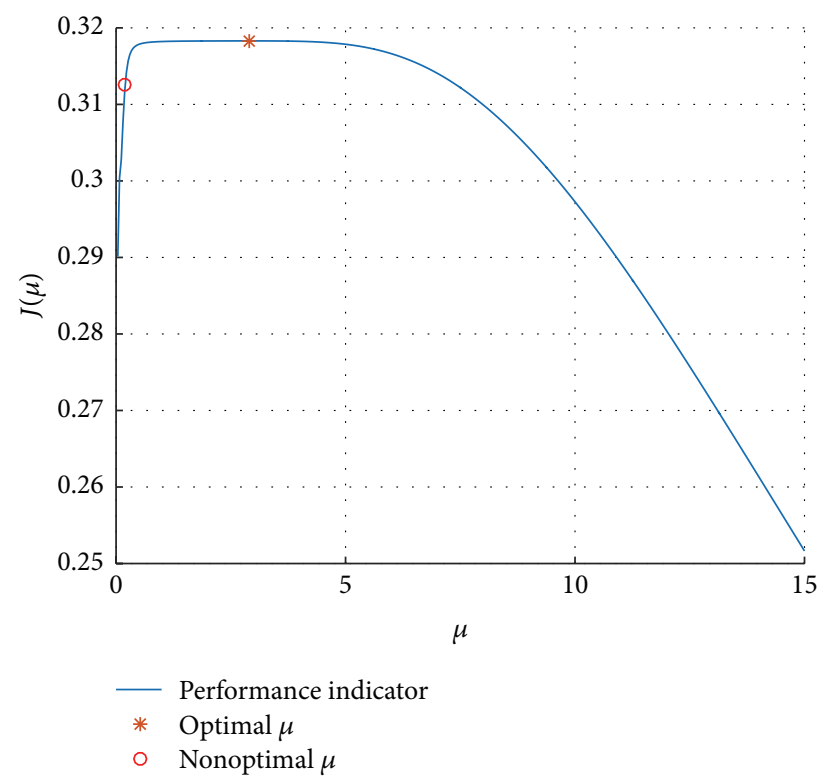

(b) Analysis of $\mu$ for $\alpha=1.5$

FIgURE 3: Analysis of $\mu$ for two values of $\alpha$.

phase response exhibits bias. It can be seen in Figure 4(c) that the difference between optimal and nonoptimal $\mu$ is on the level of $10 \%$ of optimized function value.

4.3. Optimization Problem. The third example chosen to show the use of approximation method consists of optimization of noninteger order closed-loop system. In contrast to classical integer order systems, the solution of noninteger order system yields problems with either exact form of the solution or its numerical implementation as it requires the whole "history" beginning with initial conditions (lack of semigroup property). Moreover, even though there are exact solutions presented, for example, in [26], it is worth noticing that they require special functions, that is, MittagLeffler function. Furthermore, taking into account that $s^{\alpha}$ is a multivalued function for complex $s$ and noninteger $\alpha$, we can notice that direct calculation of integral of impulse response is complicated. Therefore, an approximation method is indispensable for effective optimization.

The main purpose is to find optimal $P D^{\alpha}$ controller parameters, $\alpha, K_{D}$, and $K_{P}$, in order to efficiently bring the closed-loop system to zero. For this purpose, we chose the following objective function

$$
J=\int_{0}^{\infty} g^{2}(t) d t
$$

where $g(t)$ denotes the impulse response of the system. It can be observed (see, e.g., [21, 24]) that

$$
J=\int_{0}^{\infty} y^{2}(t) d t=\|y\|_{\mathscr{L}^{2}} \approx \sum_{i=1}^{n} \beta_{i}^{2} .
$$

Thus, the optimization consists in minimizing the performance index (77) with respect to three parameters of controller (80). It is important to notice that during the procedure both types of parameters are optimized, $\mu$ and controller parameters.

Other examples of performance indicators were analyzed, for example, in [31].

4.3.1. Optimization Algorithm. The algorithm consists of two main steps described below:

(1) Set starting points for controller parameters in such a way that the noninteger order system is asymptotically stable.

(2) Perform the optimization routine calculating the chosen performance indicator.

(a) For given values of parameters of the controller perform the stability check of the noninteger order system. If the parameters lie outside the asymptotic stability region, set the value of performance indicator to infinity and go to the third step. If the system is asymptotically stable, then go to second step.

(b) In order to approximate the stable system with LIRA method, find current optimal $\mu$ for given controller parameters. If $\mu$ is infinite in this iteration, set the performance indicator to infinity. Otherwise, calculate the value of performance indicator using the approximation method.

(c) On the basis of value of performance indicator, find the controller parameters for next step according to chosen optimization routine.

It is worth noticing that although the optimization is performed on approximated system, the stability test is 


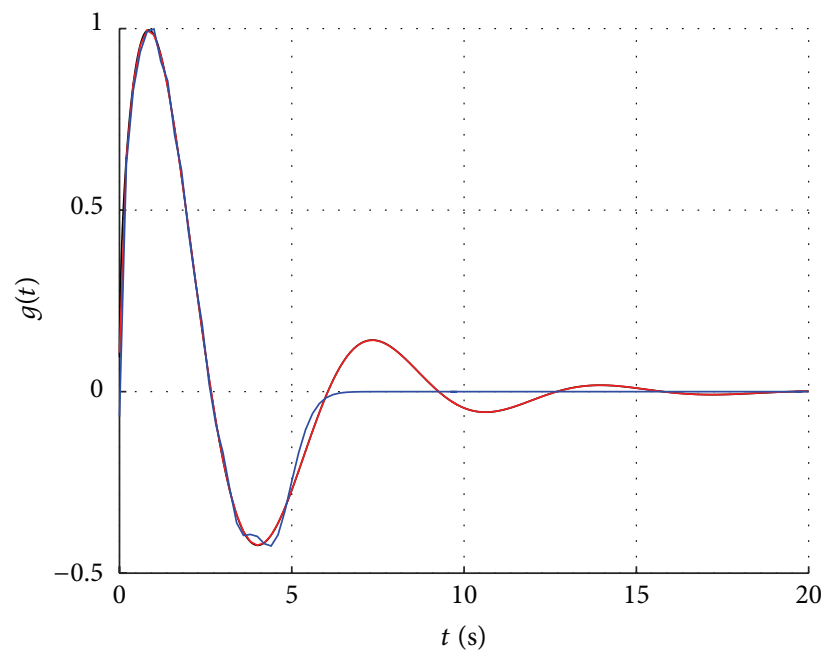

- True impulse response

- Approximation

— Nonoptimal approximation

(a) Impulse response for filter with complex poles in sector of right half plane preserving stability for $\alpha=0.7$
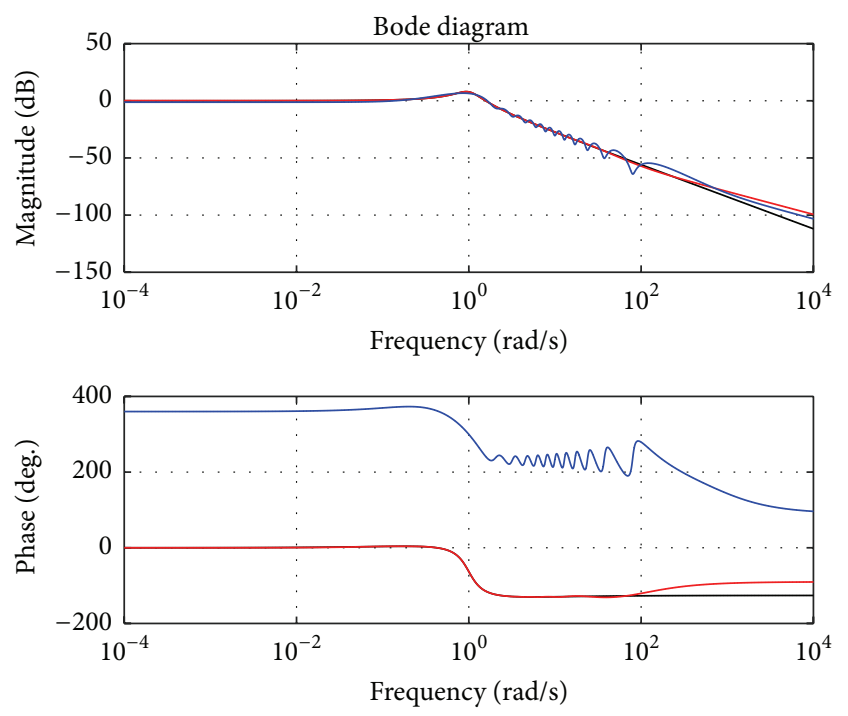

— True characteristic

- Approximation

— Nonoptimal approximation

(b) Frequency response for filter with complex poles in sector of right half plane preserving stability for $\alpha=0.7$

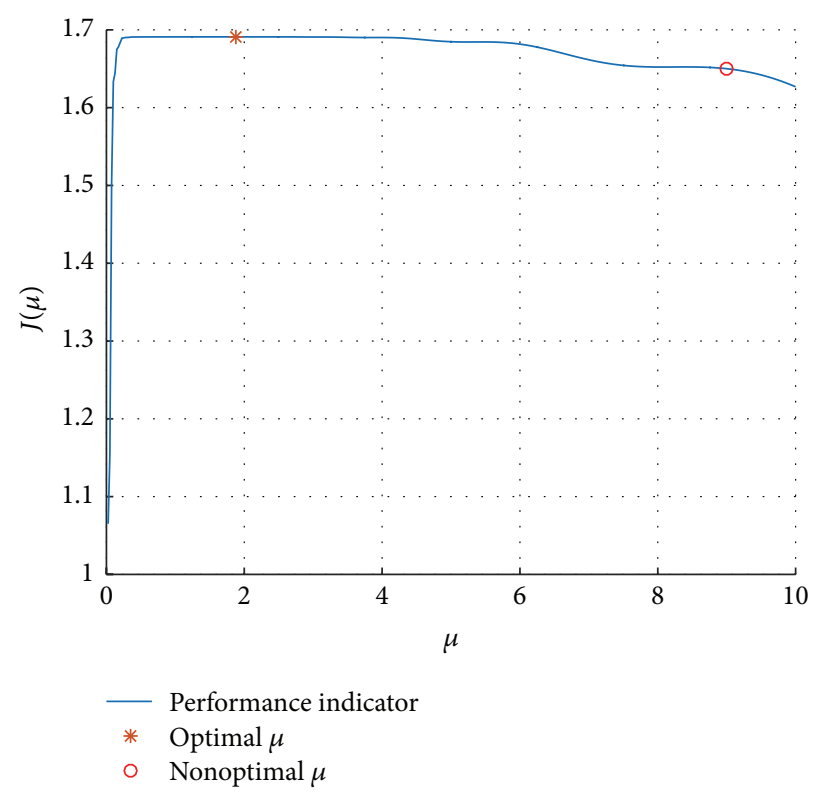

(c) Analysis of $\mu$ for filter with complex poles in sector of right half plane preserving stability for $\alpha=0.7$

FIgURE 4: Analysis for a chosen filter.

performed for noninteger order one. To verify the stability, we used an original algorithm based on $D$-partition method for noninteger order systems (see, e.g., [32]).

4.3.2. Closed-Loop System. As an example, we analyzed an integer order system from Figure 5 with transfer function

$$
G_{0}(s)=\frac{5}{s^{4}+8 s^{3}+21 s^{2}+20 s+5}
$$

and noninteger order $P D^{\alpha}$ controller

$$
G_{C}(s)=K_{P}+K_{D} s^{\alpha} .
$$

The transfer function of a closed-loop system is

$$
G(s)=\frac{5}{s^{4}+8 s^{3}+21 s^{2}+20 s+K_{D} s^{\alpha}+5+K_{P}} .
$$

The poles of system (79) are real and negative. The similar system was widely analyzed in literature (see, e.g., [33]) in 


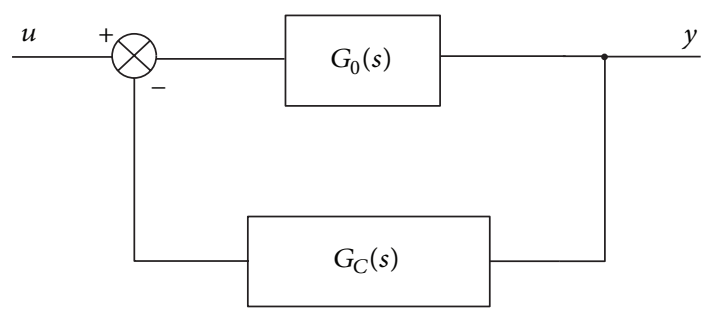

Figure 5: Closed-loop system.

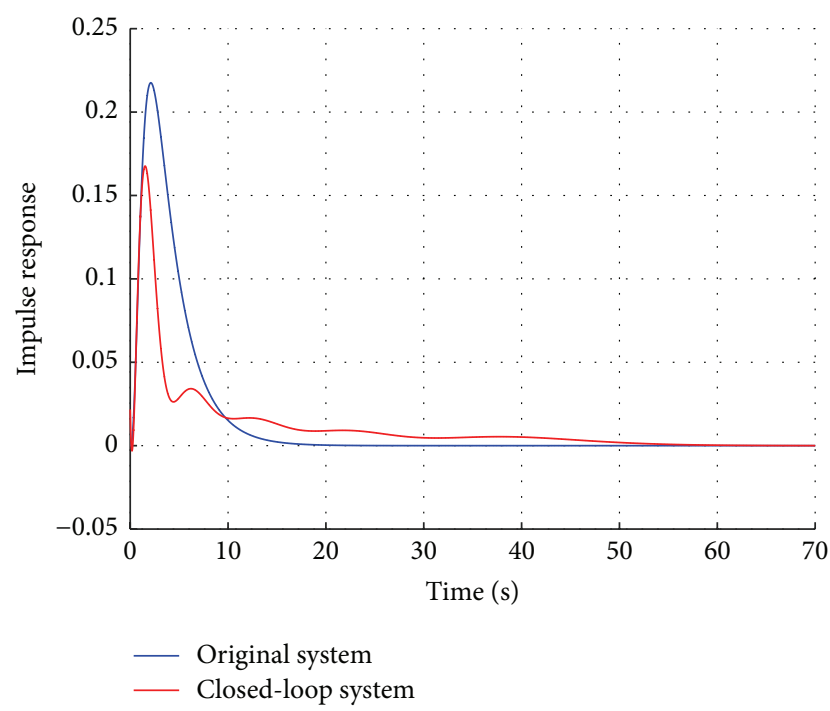

FIGURE 6: Closed-loop system impulse response compared with original system.

form of RC ladder system. Among the others, it is very useful as it allows approximating some distributed systems, for example, transmission line [34].

4.3.3. Optimization with respect to $\alpha, K_{P}$, and $K_{D}$. The system was analyzed in, for example, [22]. The parameters of approximation are highly nonlinear with respect to $\alpha$ and therefore the performance indicator is not necessarily strictly convex.

The optimal values are $\alpha=0.4467, K_{P}=-1$, and $K_{D}=16$ for $\mu=0.4378$. In Figure 6 , we depicted the results in the form of impulse response of original system and the system with the controller.

4.3.4. Analysis of the Method. The approximation method proved to be useful for tuning noninteger order controllers. It allows approximating noninteger order system with an asymptotically stable system of integer order differential equations. Therefore, it is possible to use classical theory, especially for calculation of performance indicators. Although the approximation is stable, it does not ensure stability of noninteger order system.

\section{Conclusions}

In this paper, we have shown theoretical basis and actual effectiveness of LIRA method for approximating noninteger order systems. As it can be seen (documented in earlier works, i.e., $[21-24,35])$, it is an approximation of inputoutput relationships, adequate for such purposes like filtering or control loop optimization. The main contribution of this paper over the earlier results was relaxation of the assumptions regarding the impulse response of approximated system, as it is no longer required for it to be bounded.

The main weakness of the method lies in computation of approximation coefficients $\beta_{i}$. While formula for computation of scalar products in Lemma 11 is useful for any order of approximation, it still requires computation of transfer function derivatives. The best results with respect to precision come from symbolic computation (particularly in Maple, MATLAB Symbolic toolbox gives worse results when converting from symbolic to numeric). It is, however, very time consuming, which is especially troublesome during optimization. Different approach is to use Leibnitz formula for derivative of $n$th order described in [20]; unfortunately, this formulation, for high $n$, introduces multiple factorials of high numbers which leads to numerical errors caused by factorial approximation (it was shown in [23]). Also changes in transfer function for this approach lead to rather advanced code modifications. Difference approximations of derivatives are too sensitive to be considered at all. One could consider computing scalar products directly from definition but it requires the time domain form of impulse response, which is usually either unavailable or expressed with special functions.

The other less severe problem is that there are still no definitive criteria on when an impulse response of noninteger order system is in $\mathscr{L}_{1} \cap \mathscr{L}_{2}$. The working hypothesis that is described in Remark 15 even if proved will be only a sufficient condition. It was observed in [24] that approximation can be convergent also for relative degrees less than one.

Further development of the method will include addressing the mentioned flaws. However, the main interest of the authors is in applications. At the moment, we work on EEG signal filtering, vibration signal filtering, optimization of noninteger order controllers, and development of circuit realizations of noninteger order systems.

\section{Competing Interests}

The authors declare that they have no competing interests.

\section{Acknowledgments}

The work is realized in the scope of project titled "Design and Application of Non-Integer Order Subsystems in Control Systems." Project was financed by National Science Centre on the base of Decision no. DEC-2013/09/D/ST7/03960.

\section{References}

[1] A. Oustaloup, La Commande CRONE: Commande Robuste d'Ordre Non Entier, Hermès, Paris, France, 1991. 
[2] A. Oustaloup, F. Levron, B. Mathieu, and F. M. Nanot, "Frequency-band complex noninteger differentiator: characterization and synthesis," IEEE Transactions on Circuits and Systems I: Fundamental Theory and Applications, vol. 47, no. 1, pp. 25-39, 2000.

[3] Y. Chen, B. M. Vinagre, and I. Podlubny, "Continued fraction expansion approaches to discretizing fractional order derivatives-an expository review," Nonlinear Dynamics, vol. 38, no. 1-4, pp. 155-170, 2004.

[4] B. M. Vinagre, I. Podlubny, A. Hernandez, and V. Feliu, "Some approximations of fractional order operators used in control theory and applications," Fractional Calculus and Applied Analysis, vol. 3, no. 3, pp. 231-248, 2000.

[5] B. M. Vinagre, Y. Q. Chen, and I. Petráś, "Two direct tustin discretization methods for fractional-order differentiator/integrator," Journal of the Franklin Institute, vol. 340, no. 5, pp. 349-362, 2003.

[6] C. A. Monje, Y. Chen, B. M. Vinagre, D. Xue, and V. FeliuBatlle, Fractional-Order Systems and Controls: Fundamentals and Applications, Advances in Industrial Control, Springer, London, UK, 2010.

[7] J. Baranowski, W. Bauer, and M. Zagórowska, "Stability properties of discrete time-domain oustaloup approximation," in Theoretical Developments and Applications of Non-Integer Order Systems, S. Domek and P. Dworak, Eds., vol. 357 of Lecture Notes in Electrical Engineering, pp. 93-103, Springer, Berlin, Germany, 2016.

[8] J. Baranowski, W. Bauer, M. Zagórowska, T. Dziwinski, and P. Piatek, "Time-domain oustaloup approximation," in Proceedings of the 20th International Conference on Methods and Models in Automation and Robotics (MMAR '15), pp. 116-120, IEEE, Miedzyzdroje, Poland, August 2015.

[9] C.-C. Tseng, "Design of FIR and IIR fractional order simpson digital integrators," Signal Processing, vol. 87, no. 5, pp. 10451057, 2007.

[10] Y. Ferdi, "Computation of fractional order derivative and integral via power series expansion and signal modelling," Nonlinear Dynamics, vol. 46, no. 1-2, pp. 1-15, 2006.

[11] F. Leulmi and Y. Ferdi, "Improved digital rational approximation of the operator $S^{\alpha}$ using second-order s-to-z transform and signal modeling," Circuits, Systems, and Signal Processing, vol. 34, no. 6, pp. 1869-1891, 2015.

[12] G. Montseny, "Diffusive representation of pseudo-differential time-operators," ESAIM: Proceedings, vol. 5, pp. 159-175, 1998.

[13] D. Heleschewitz and D. Matignon, "Diffusive realisations of fractional integrodifferential operators: structural analysis under approximation," in Proceedings of the IFAC Conference Systems Structure and Control, vol. 2, pp. 243-248, Nantes, France, 1998.

[14] S. Liang, C. Peng, Z. Liao, and Y. Wang, "State space approximation for general fractional order dynamic systems," International Journal of Systems Science, vol. 45, no. 10, pp. 2203-2212, 2014.

[15] J. C. Trigeassou, N. Maamri, J. Sabatier, and A. Oustaloup, "State variables and transients of fractional order differential systems," Computers \& Mathematics with Applications, vol. 64, no. 10, pp. 3117-3140, 2012.

[16] Y. Wei, S. Cheng, Y. Hu, and Y. Wang, "Fractional order adaptive backstepping control based on frequency distributed model," in Proceedings of the 54th IEEE Conference on Decision and Control (CDC '15), pp. 7616-7621, Osaka, Japan, December 2015.
[17] P. Grabowski, "Stabilization of wave equation using standard/fractional derivative in boundary damping," in Advances in the Theory and Applications of Non-Integer Order Systems, W. Mitkowski, J. Kacprzyk, and J. Baranowski, Eds., vol. 257 of Lecture Notes in Electrical Engineering, pp. 101-121, Springer, Heidelberg, Germany, 2013.

[18] M. Aoun, R. Malti, F. Levron, and A. Oustaloup, "Synthesis of fractional Laguerre basis for system approximation," Automatica, vol. 43, no. 9, pp. 1640-1648, 2007.

[19] G. Maione, "Laguerre approximation of fractional systems," Electronics Letters, vol. 38, no. 20, pp. 1234-1236, 2002.

[20] P. Bania and J. Baranowski, "Laguerre polynomial approximation of fractional order linear systems," in Advances in the Theory and Applications of Non-Integer Order Systems: 5th Conference on Non-Integer Order Calculus and Its Applications, Cracow, Poland, vol. 257 of Lecture Notes in Electrical Engineering, pp. 171-182, Springer, Cham, Switzerland, 2013.

[21] M. Zagórowska, "Parametric optimization of non-integer order $\mathrm{PD}^{\mu}$ controller for delayed system," in Theoretical Developments and Applications of Non-Integer Order Systems, pp. 259-270, Springer, New York, NY, USA, 2016.

[22] M. Zagórowska, J. Baranowski, P. Bania, W. Bauer, T. Dziwinski, and P. Piatek, "Parametric optimization of pd controller using laguerre approximation," in Proceedings of the 20th International Conference on Methods and Models in Automation and Robotics (MMAR '15), pp. 104-109, IEEE, Międzyzdroje, Poland, August 2015.

[23] M. Zagórowska, J. Baranowski, P. Bania, P. Piątek, W. Bauer, and T. Dziwiński, "Impulse response approximation method for 'fractional order lag"', in Advances in Modelling and Control of Non-Integer-Order Systems, K. J. Latawiec, M. Łukaniszyn, and R. Stanisławski, Eds., vol. 320 of Lecture Notes in Electrical Engineering, pp. 113-122, Springer, 2015.

[24] J. Baranowski, W. Bauer, M. Zagórowska, and P. Piatek, “On digital realizations of non-integer order filters," Circuits, Systems, and Signal Processing, vol. 35, no. 6, pp. 2083-2107, 2016.

[25] P. Grabowski, Lecture Notes on Optimal Control Systems, Wydawnictwa AGH, Kraków, Poland, 1999, http://home .agh.edu.pl/ pgrab/grabowski_files/lecturedition2/newlecture .xml.

[26] I. Podlubny, Fractional Differential Equations: An Introduction to Fractional Derivatives, Fractional Differential Equations, to Methods of Their Solution and Some of Their Applications, Mathematics in Science and Engineering, Elsevier Science, 1999.

[27] A. G. Radwan, A. S. Elwakil, and A. M. Soliman, "On the generalization of second-order filters to the fractional-order domain," Journal of Circuits, Systems and Computers, vol. 18, no. 2, pp. 361-386, 2009.

[28] J. Baranowski, P. Piatek, W. Bauer, T. Dziwinski, and M. Zagórowska, "Bi-fractional filters, part 2: right half-plane case," in Proceedings of the 19th International Conference on Methods and Models in Automation and Robotics (MMAR '14), pp. 369373, IEEE, 2014.

[29] P. Piątek, J. Baranowski, M. Zagórowska, W. Bauer, and T. Dziwiński, "Bi-fractional filters, part 1: Left half-plane case," in Advances in Modelling and Control of Non-Integer-Order Systems, vol. 320 of Lecture Notes in Electrical Engineering, pp. 81-90, Springer, 2015.

[30] T. Kaczorek, Selected Problems of Fractional Systems Theory, vol. 411 of Lecture Notes in Control and Information Sciences, Springer, Berlin, Germany, 2011. 
[31] M. Zagórowska, "Analysis of performance indicators for optimization of non-integer order controllers," in Proceedings of the 39th International Conference on Telecommunications and Signal Processing, 2016.

[32] A. Ruszewski, "Stability regions of closed loop system with time delay inertial plant of fractional order and fractional order PI controller," Bulletin of the Polish Academy of Sciences: Technical Sciences, vol. 56, no. 4, 2008.

[33] W. Mitkowski, Stabilizacja Systemów Dynamicznych, Wydawnictwa AGH, Kraków, Poland, 1996.

[34] W. Mitkowski, "Remarks about energy transfer in an RC ladder network," International Journal of Applied Mathematics and Computer Science, vol. 13, no. 2, pp. 193-198, 2003.

[35] J. Baranowski, M. Zagórowska, P. Bania, W. Bauer, T. Dziwiński, and P. Piątek, "Impulse response approximation method for bi-fractional filter," in Proceedings of the 19th International Conference on Methods and Models in Automation and Robotics (MMAR '14), pp. 379-383, IEEE, Miedzyzdroje, Poland, September 2014. 


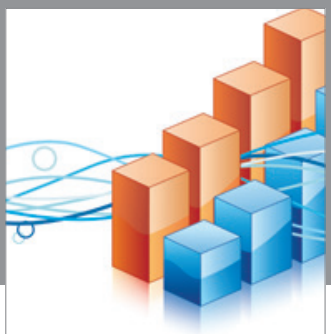

Advances in

Operations Research

vatem alat4

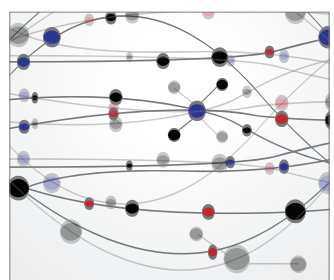

\section{The Scientific} World Journal
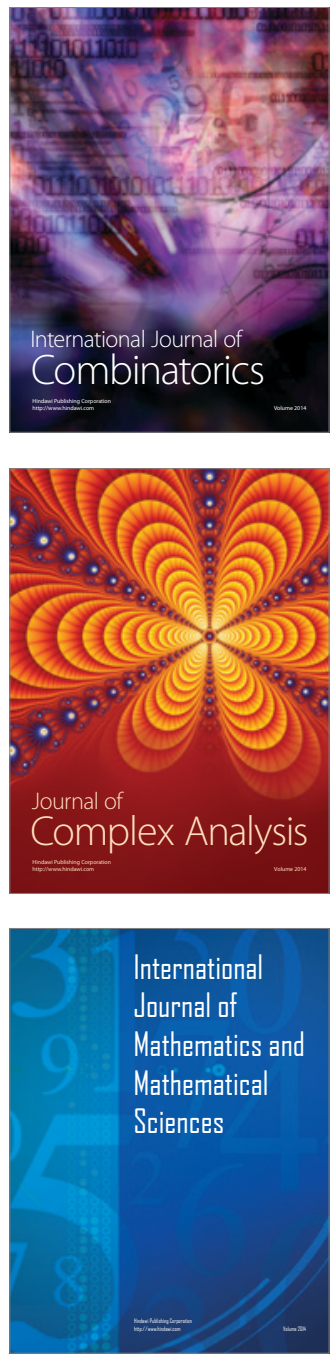
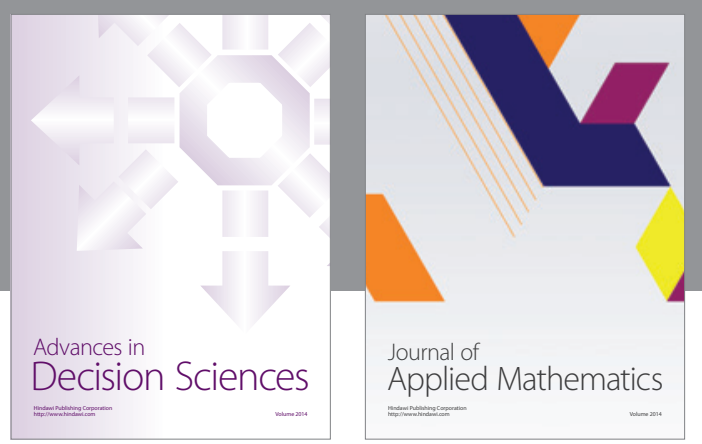

Algebra

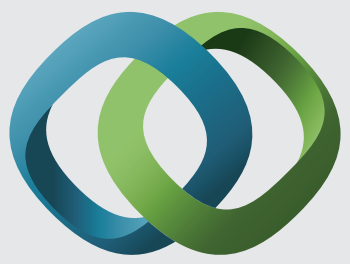

\section{Hindawi}

Submit your manuscripts at

http://www.hindawi.com
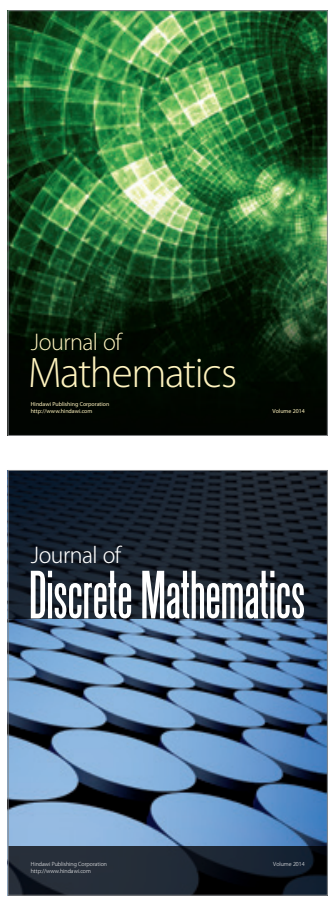

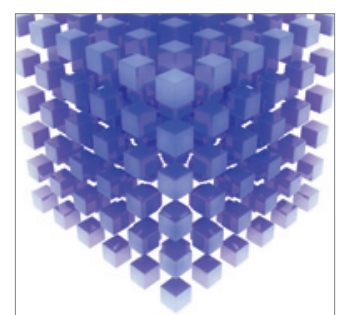

Mathematical Problems in Engineering
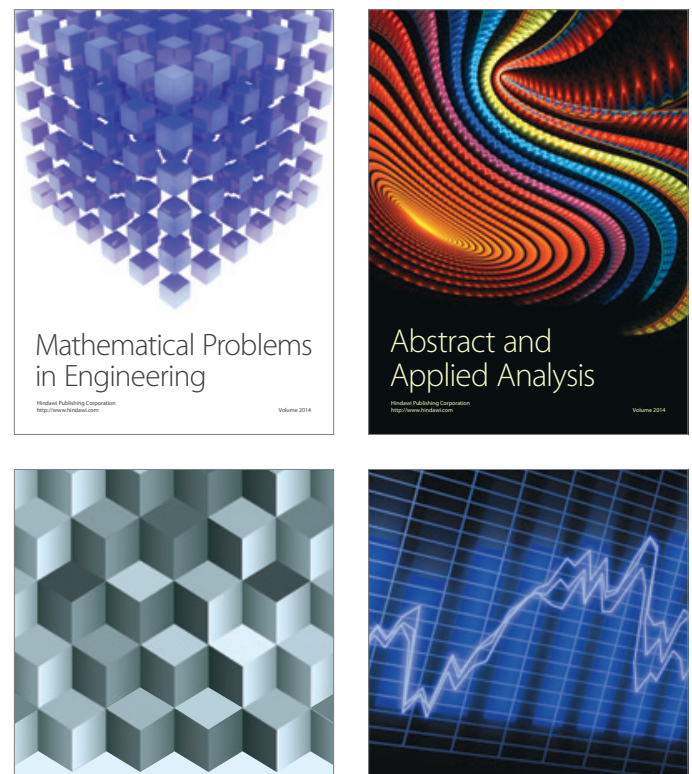

Journal of

Function Spaces

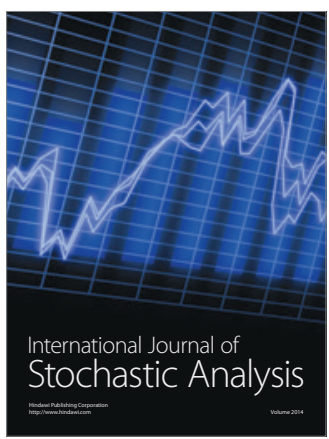

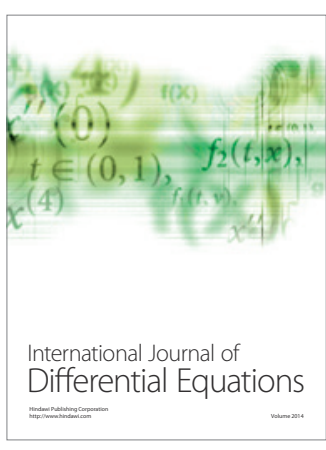
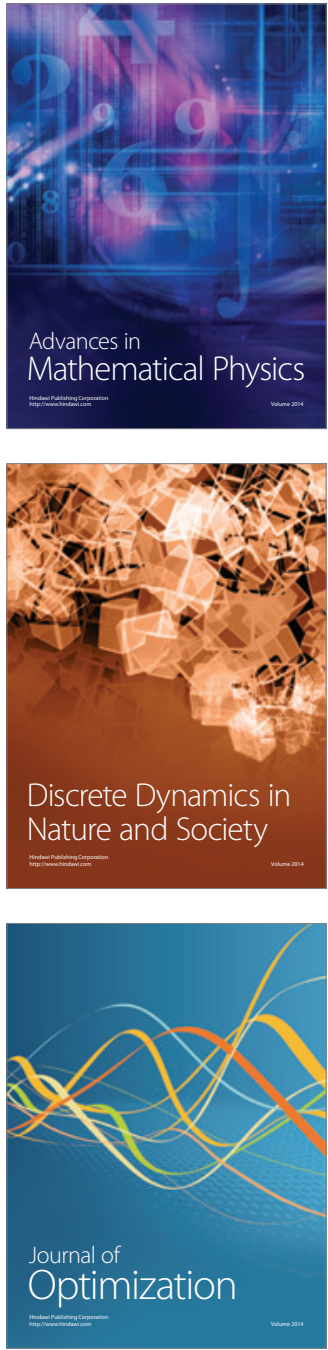